\title{
The Significance of Friend Leukemia Virus Integration 1 Transcription Factor (Fli-1) in Astrocytoma
}

\author{
Hala A. Agina, Ahlam A. Abdelmaksoud, Eman S. Omar, Marwa S. Abdallah, Shimaa G. \\ Ahmed
}

Department of Pathology, Faculty of Medicine Benha University, Egypt.

\section{Correspondence to:}

Shimaa G. Ahmed, Department of Pathology, Faculty of Medicine Benha University, Egypt.

\section{Email:}

shimaagama190@yahoo.com

Received: 30 August 2021

Accepted: 15February 2022

\begin{abstract}
Background: Astrocytoma is the most common type of glioma. Fli-1 is a nuclear transcription factor that increase cellular proliferation and tumorigenesis providing a prognostic tool for many human tumors. Therefore, Fli- 1 may have a role in astrocytoma. Aim: To study the expression of Fli-1 in astrocytoma and its correlation to clinicopathological aspects. Subjects and
\end{abstract} methods: This is a retrospective study performed upon 45 cases grouped as $22.2 \%$ of grade I astrocytoma, $26.7 \%$ of grade II astrocytoma, $13.3 \%$ of grade III astrocytoma and $37.8 \%$ of grade IV astrocytoma. Immunohistochemical staining of Fli-1 antibody was applied on formalin-fixed, paraffin-embedded blocks. The correlation between Fli-1 expression and clinicopathologic parameters was statistically studied. Results: High expression of Fli -1 was seen in $60 \%$ of astrocytoma, while low expression was seen in $40 \%$ of astrocytoma cases. Fli -1 expression showed highly significant associations with age, tumor size, tumor site, tumor grade, tumor type, progression free and overall survivals of studied cases ( $\mathrm{P}$ value $<0.01$ ). There was significant correlation between the Fli-1expression and recurrence $(\mathrm{P}$ value $<0.05)$. No significant correlations were found between Fli-1 expression and sex or type of biopsy in studied cases ( $p$ value $>0.05$ ). Conclusion: Fli- 1 score $>2$ is better to discriminate between low and high grade astrocytoma. Fli-1 score can be diagnostic to differentiate between low and high grade astrocytoma. Fli-1 may be a prognostic marker in astrocytoma to predict recurrence \& patient survival.

Keywords: Fli-1, Astrocytoma, prognostic. 


\section{Introduction}

Gliomas are the most common intracranial neoplasms representing $27.7 \%$ of all brain tumors and $82.3 \%$ of malignant brain tumors. Astrocytoma is the most common type of glioma, representing $64 \%$ of human Central nervous system (CNS) malignancies (1).

In Egypt, gliomas account for $37.3 \%$ of primary CNS tumors. Astrocytic tumors are the most common CNS tumors and the most common glial tumors accounts for $79.4 \%$ of all gliomas (2, 3). Environmental risk factors as radiation, infectious and chemical agents, genetic risk factors are thought to increase chance of this tumor (4).

Fli-1 is encoded by the Fli-1 gene, a protooncogene and features a 98-amino-acid DNA binding domain (5). Fli-1 is a member of the E26 transformation specific family (ETS), transcription factor family. ETS transcription factors regulate the expression of oncogenes, tumor suppressor genes, and other genes related to vessel formation, invasion, and metastasis (6).

The nuclear transcription factor Fli-1 has been shown to increase cellular proliferation and tumorigenesis in many types of cancers including melanoma (7), nasopharyngeal carcinoma (NPC) (8), breast

cancer (9), ovarian cancer (5), endometrial cancer (10) and non-small cell lung carcinoma (NSCLC) (11).

This study aimed at assessment of immunohistochemical expression of Fli-1 in astrocytoma, its clinico-pathological correlation.

\section{Material and Methods}

This study was a retrospective, controlled, selective study performed upon formalinfixed, paraffin embedded blocks of selected 45 cases of Egyptian astrocytoma patients .The study was performed in Pathology Department; Benha Faculty of Medicine. Cases were processed during the years from 2013 to 2016 . The study was approved by the Ethical committee of faculty of Medicine, Benha University

Clinicopathological data were collected from the files of the patients including patients' demographic, clinical and histopathological data as patient's age, sex, tumor site, tumor size, tumor grade, tumor type, type of biopsy, recurrence ,overall and progression free survivals. Cases were classified into 5 groups according to age and 2 groups according to size (6). 


\section{Histopathological study}

Paraffin blocks were collected and two slides of each block of 3 micron thickness were cut, one on plain slide and the other on positively charged slide. The sections were dewaxed at 56 o $\mathrm{C}$ for 2 hours and one slide was made ready for staining with hematoxylin and eosin. Slides of all cases were reviewed by two observers simultaneously to confirm the diagnosis. The remarkable histopathological data such as tumor grade were noted. Astrocytoma cases were classified and graded as stated in the WHO classification 2016.Grade I including pilocytic and subependymal giant cell astrocytoma. Grade II including diffuse astrocytoma and pleomorphic xanthoastrocytoma, Grade III including anaplastic astrocytoma, Grade IV astrocytoma including glioblastoma.

\section{Immunohistochemical study}

Slides were immune stained using a standard labeled streptavidin-biotin system (Genemed, CA 94080, USA, South San Francisco) with Fli-1 polyclonal antibody (Chongqing Biospes Co., Ltd, China) at a dilution of 1:50, at room temperature overnight. Immunodetection was carried out using detection kits (Dako, Glostrup, Denmark). It was performed based on manufacturer's instructions. Antigen retrieval was done by using $10 \mathrm{mmol} / \mathrm{L}$ citrate monohydrate buffer (PH 6.0) and heated for 20 minutes in the microwave. DAB) was used as chromogen. Normal tonsillar tissue was used as external positive control. Negative control was obtained by processing tissue section with omitting the primary antibody and adding Phosphate Buffered Saline (PBS) instead.

\section{Immunostaining evaluation:}

Positivity was considered as brownish nuclear staining of tumor cells. The results of immunohistochemical staining was scored on a scale of 0 to 9 . The percentage of positive tumor cells was classified as: 0: no positive tumor cells, 1: less than $10 \%$ positive tumor cells, $2: \quad 10-50 \%$ positive cells, 3: more than $50 \%$ positive cells. The staining intensity was classified as: 0: no staining, 1: weak staining, 2: moderate staining, 3: strong staining. The Fli-1 score was calculated by multiplying the intensity and percentage of positive tumor cells in each sample to yield possible scores of 0,1 , 2, 3, 4, 6, and 9. Immunohistochemical staining results were classified as low-level expression and high-level expression (low and high Fli-1 Scores). A total score of 4 was set as a cut-off; so $\geq 4$ was considered 
high Fli-1 score and $<3$ considered low Fli-1 score (6).

Statistical analysis: Results were analyzed using SPSS (version 16) statistical package for Microsoft windows (SPSS Inc., Chicago, IL, USA). Categorical data were expressed as numbers and percentages. Numerical data were expressed as mean \pm standard deviation. Pearson Chi square test, One Way ANOVA tests and Fisher`s Exact test (FET) were used to assess correlations between groups. P-value $>0.05$ was considered non-significant (NS), $\mathrm{p}$ value $<0.05$ was considered significant (S). $\mathrm{P}$ value $<0.01$ was considered highly significant (HS).

Receiver operator characteristic (ROC) curve was also used to determine cut off value of Fli-1 marker and other parameters. Survival analysis was done using a KaplanMeier analysis and subsequent log-rank analysis to confirm the correlation between Fli-1 expression, grade of tumor, size of tumor and survival (overall and progression free survival) in astrocytoma patients.

\section{Results:}

\section{Clinicopathological features:}

The examined 45 cases were 10 cases (22.2 $\%$ ) of grade I astrocytoma (8 pilocytic astrocytoma cases and 2 subependymal giant cell astrocytoma cases), 13 cases (26.7\%) of grade II astrocytoma (9 diffuse astrocytoma cases and 4 pleomorphic xanthoastrocytoma cases), 6 cases (13.3\%) of grade III astrocytoma (anaplastic astrocytoma) and 16 cases (37.8\%) of grade IV astrocytoma (glioblastoma).

\section{Immunohistochemical results:}

Fli-1 showed nuclear expression in astrocytoma cells. The percentage and intensity of Fli-1 in different study groups were studied and Fli-1 score was calculated. Out of 45 cases of astrocytoma, 27 cases (60\%) showed high Fli -1 score (Figure $1,2)$ and 18 cases $(40 \%)$ showed low Fli-1 score (Figure 3, 4). All grade III, IV cases had high Fli-1 score, while $21.7 \%$ of grade I, II cases had high Fli-1 score. Fli-1 expression was related with different clinicopathological findings (Table 1).

There was highly significant statistical association between Fli -1 expression and age, tumor size, tumor site, tumor grade, tumor_type, progression free, overall survivals of studied cases ( $\mathrm{P}$ value $<0.01$ ). The results revealed significant correlations between the expression levels of Fli-1 and recurrence ( $\mathrm{P}$ value <0.05). However, no significant correlations were found between 
Fli-1 expression and sex or type of biopsy in studied cases ( $\mathrm{p}$ value $>0.05$ ).

\section{Receiver operating characteristic curve (ROC curve) of Fli-1 as a predictor}

\section{between low grade and high grade of tumor}

The Receiver operating characteristic curve (ROC) curve showed that the cutoff point $>2$ for Fli-1 score to differentiate between high grade and low grade astrocytoma showed sensitivity of $95.65 \%$, specificity of $77.27 \%$ and area under curve (AUC) of $90.8 \%$ while the cutoff point $>4$ showed sensitivity of $81.5 \%$, specificity of
94.4\% and AUC of $86.7 \%$ (Table 2)

(Graph 1).

\section{Kaplan Mayer analysis}

Kaplan Mayer analysis showed that there was statistically significant increase in the mean overall survival in cases with low Fli1 score than those with high Fli-1 score with p-value <0. 01 (Table 3) (Graph 2).

Kaplan Mayer analysis showed that there was statistically significant increase in the progression free survival in cases with low Fli-1 score than those with high Fli-1 score with p-value <0.01 (Table 4$)($ Graph 3 ).

Table 1: Correlation between Fli-1 and other clinicopathological parameters.

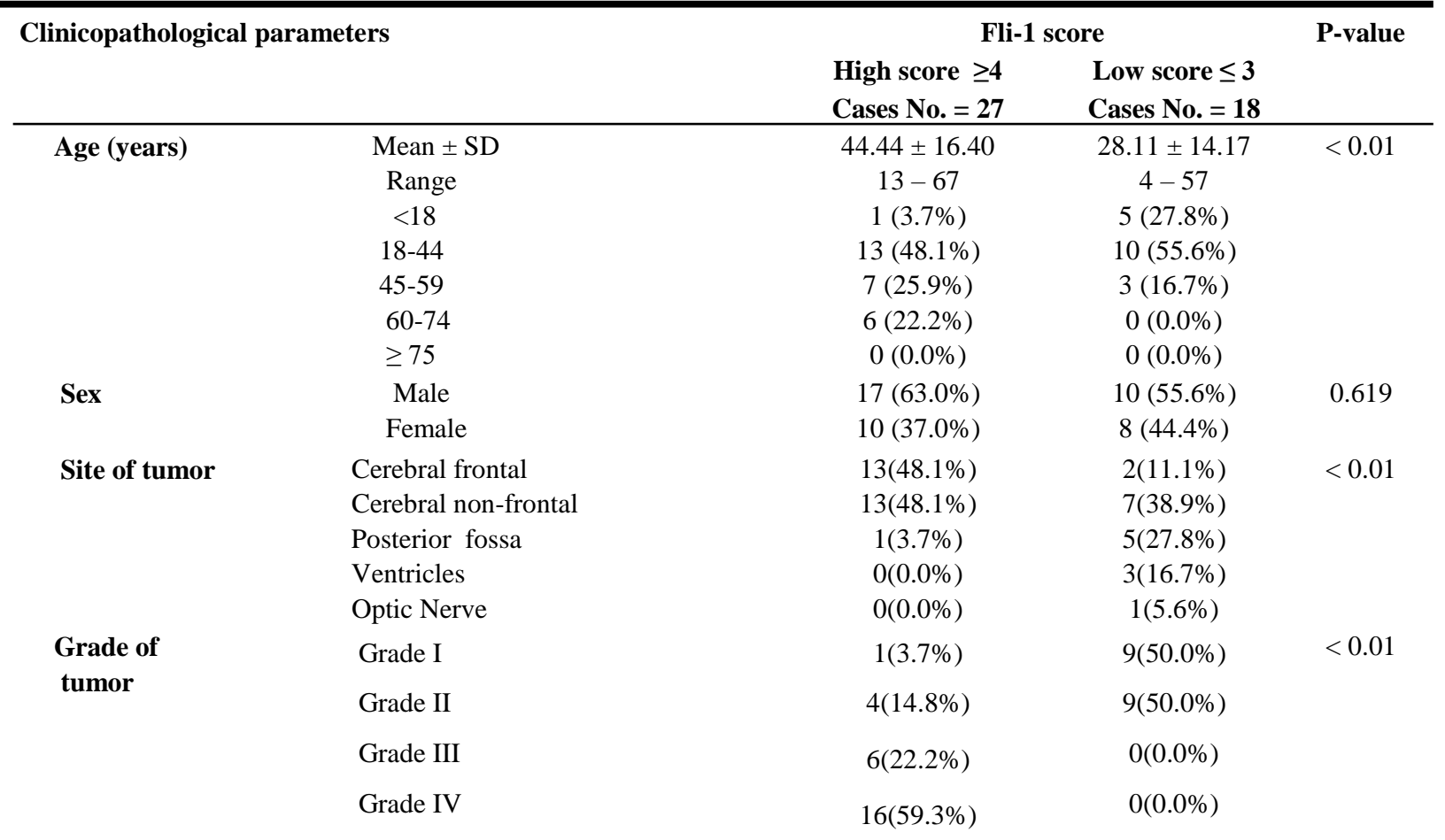


Benha medical journal, vol. 39, Special issue (academic), 2022

\begin{tabular}{|c|c|c|c|c|}
\hline \multirow{7}{*}{$\begin{array}{l}\text { Type of } \\
\text { tumor }\end{array}$} & Glioblastoma & $16(59.3 \%)$ & $0(0 \%)$ & $<0.01$ \\
\hline & Anaplastic astrocytoma & $6(22.3 \%)$ & $0(0 \%)$ & \\
\hline & Diffuse astrocytoma & $3(11 \%)$ & $6(33.3 \%)$ & \\
\hline & Pleomorphic xanthoastrocytoma & $1(3.7 \%)$ & $3(16.7 \%)$ & \\
\hline & Pilocytic astrocytoma & & & \\
\hline & Subependymal giant cell & $1(3.7 \%)$ & $7(38.9 \%)$ & \\
\hline & astrocytoma & $0(0 \%)$ & $2(11 \%)$ & \\
\hline \multirow[t]{4}{*}{ Size of tumor } & Mean \pm SD & $4.83 \pm 3.09$ & $2.78 \pm 1.18$ & $<0.05$ \\
\hline & Range & $1.5-16$ & $1-5.5$ & \\
\hline & $\geq 3 \mathrm{~cm}$ & $20(74.1 \%)$ & $7(38.9 \%)$ & \\
\hline & $<3 \mathrm{~cm}$ & $7(25.9 \%)$ & $11(61.1 \%)$ & \\
\hline \multirow[t]{2}{*}{$\begin{array}{r}\text { Type of } \\
\text { biopsy }\end{array}$} & Total Excision & $22(81.5 \%)$ & $17(94.4 \%)$ & 0.210 \\
\hline & Partial Excision & $5(18.5 \%)$ & $1(5.6 \%)$ & \\
\hline \multirow[t]{2}{*}{ Recurrence } & Yes & $14(51.9 \%)$ & $3(16.7 \%)$ & $<0.05$ \\
\hline & No & $13(48.1 \%)$ & $15(83.3 \%)$ & \\
\hline \multirow{6}{*}{$\begin{array}{l}\text { Progression free } \\
\text { survival(months) }\end{array}$} & $1-6$ & $1(3.7 \%)$ & $0(0.0 \%)$ & $<0.01$ \\
\hline & $7-12$ & $11(40.7 \%)$ & $1(5.6 \%)$ & \\
\hline & $13-24$ & $11(40.7 \%)$ & $6(33.3 \%)$ & \\
\hline & $25-36$ & $3(11.1 \%)$ & $3(16.7 \%)$ & \\
\hline & $37-48$ & $1(3.7 \%)$ & $8(44.4 \%)$ & \\
\hline & $49-60$ & $0(0.0 \%)$ & $0(0.0 \%)$ & \\
\hline \multirow{4}{*}{$\begin{array}{l}\text { Overall survival } \\
\text { (months) }\end{array}$} & $13-24$ & $13(48.1 \%)$ & $0(0.0 \%)$ & $<0.01$ \\
\hline & $25-36$ & $9(33.3 \%)$ & $1(5.6 \%)$ & \\
\hline & $37-48$ & $2(7.4 \%)$ & $0(0.0 \%)$ & \\
\hline & $49-60$ & $3(11.1 \%)$ & $17(94.4 \%)$ & \\
\hline \multirow[t]{2}{*}{ Patient Outcome } & Live & $9(33.3 \%)$ & $17(94.4 \%)$ & $<0.01$ \\
\hline & Died & $18(66.7 \%)$ & $1(5.6 \%)$ & \\
\hline
\end{tabular}

P-value >0.05: Non significant (NS); P-value <0.05: Significant (S); P-value< 0.01: highly significant (HS).

Table 2: Receiver operating characteristic curve (ROC) for Fli-1 score to differentiate between low and high grade of astrocytoma tumor.

\begin{tabular}{rcccccc}
\hline \multicolumn{1}{c}{ Parameter } & AUC & Cut of Point & Sensitivity & Specificity & PPV & NPV \\
\hline Fli-1 (quantitative) & 0.908 & $>2$ & 95.65 & 77.27 & 81.5 & 94.4 \\
Fli-1 (qualitative) & 0.867 & $>4$ & $81.5 \%$ & $94.4 \%$ & $95.6 \%$ & $77.3 \%$ \\
\hline
\end{tabular}


Table 3: Kaplan Mayer analysis comparing between cases with high score of Fli-1 and those with low score of Fli-1 regarding overall survival

\begin{tabular}{cccccccccccccc}
\hline & \multicolumn{3}{c}{ OS (months) } & \multicolumn{3}{c}{ 95\% CI } & \multicolumn{4}{c}{ Survival at } & \multicolumn{3}{c}{ Log Rank Test } \\
& Mean & SE & Lower & Upper & 6 months & 1 year & 2 years & 3 years & 4 years & 5 years & X & P-value & Sig. \\
\hline High score & 32.362 & 3.065 & 26.355 & 38.369 & $100.0 \%$ & $100.0 \%$ & $63.2 \%$ & $24.1 \%$ & $12.0 \%$ & $0.0 \%$ & 26.084 & $<0.01$ & HS \\
Low score & 60.0 & 0 & 60 & 60 & $100.0 \%$ & $100.0 \%$ & $100.0 \%$ & $100.0 \%$ & $100.0 \%$ & $90.0 \%$ & & & \\
\hline
\end{tabular}

P-value >0.05: Non significant (NS); P-value <0.05: Significant (S); P-value< 0.01: highly significant (HS)

Table 4: Kaplan Mayer analysis for comparing between cases with high score of Fli-1 and those with low score of Fli-1 regarding progression free survival (months).

\begin{tabular}{lccccccccccccc}
\hline & \multicolumn{3}{c}{ PFS (months) } & \multicolumn{2}{c}{ 95\% CI } & \multicolumn{3}{c}{ Survival at } & \multicolumn{4}{c}{ Log Rank Test } \\
& Mean & SE & Lower & Upper & 6 months & 1 year & 2 years & 3 years & 4 years & 5 years & X $^{2}$ & P-value & Sig. \\
\hline High score & 31.044 & 3.539 & 24.108 & 37.981 & $96.3 \%$ & $63.0 \%$ & $50.4 \%$ & $0.0 \%$ & $0.0 \%$ & $0.0 \%$ & \\
Low score & 52.533 & 3.957 & 44.778 & 60.289 & $100.0 \%$ & $88.9 \%$ & $83.0 \%$ & $83.0 \%$ & $83.0 \%$ & $83.0 \%$ & & \\
\hline
\end{tabular}

P-value >0.05: Non significant (NS); P-value <0.05: Significant (S); P-value< 0.01: highly significant (HS).

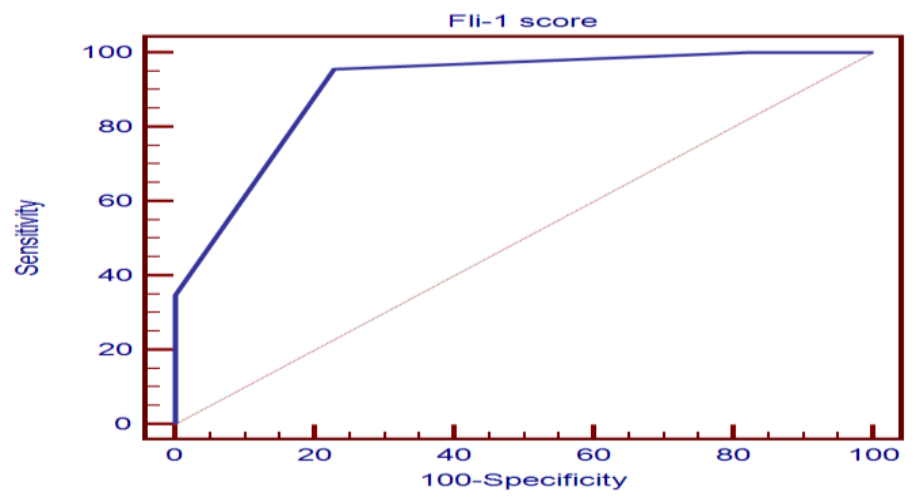

Graph 1: Roc curve for Fli-1 score. 
Benha medical journal, vol. 39, Special issue (academic), 2022

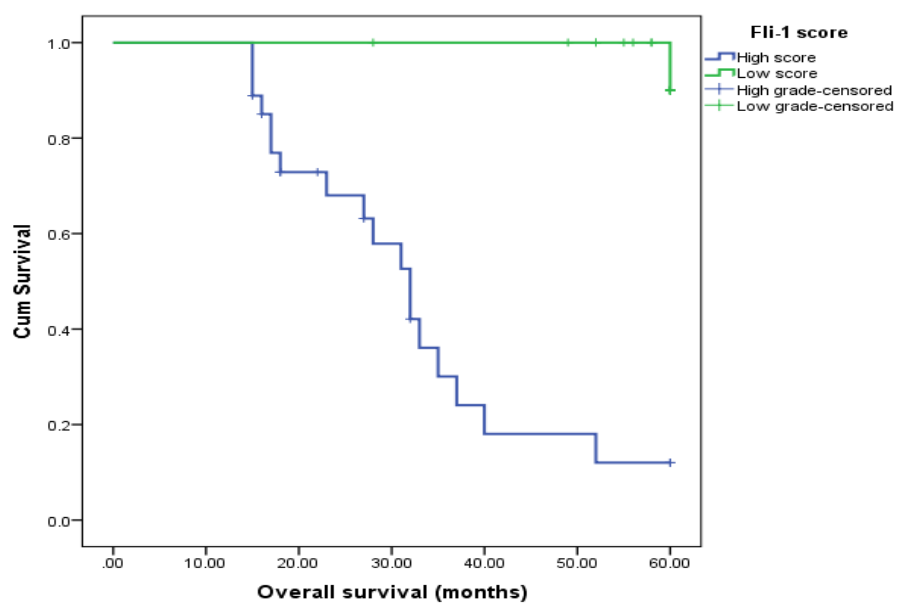

Graph 2: Kaplan Mayer curve for Comparing between cases with high score of Fli-1 and those with low score of Fli-1 regarding overall survival.

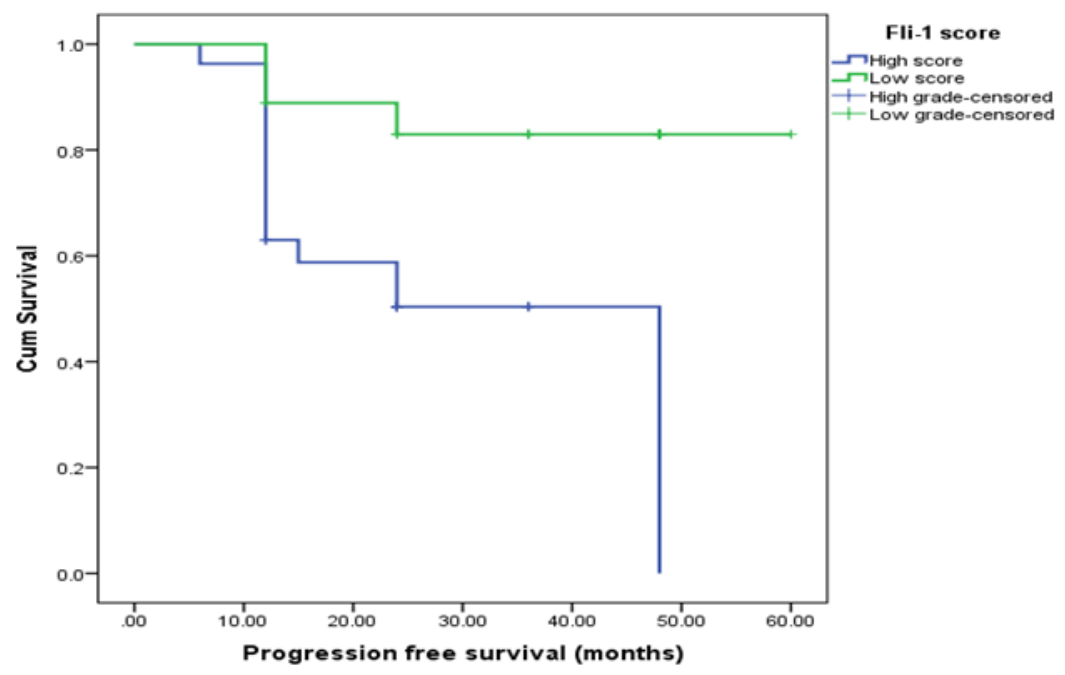

Graph 3: Kaplan Mayer curve for Comparing between cases with high score of Fli-1 and those with low score of Fli-1 regarding progression free survival. 


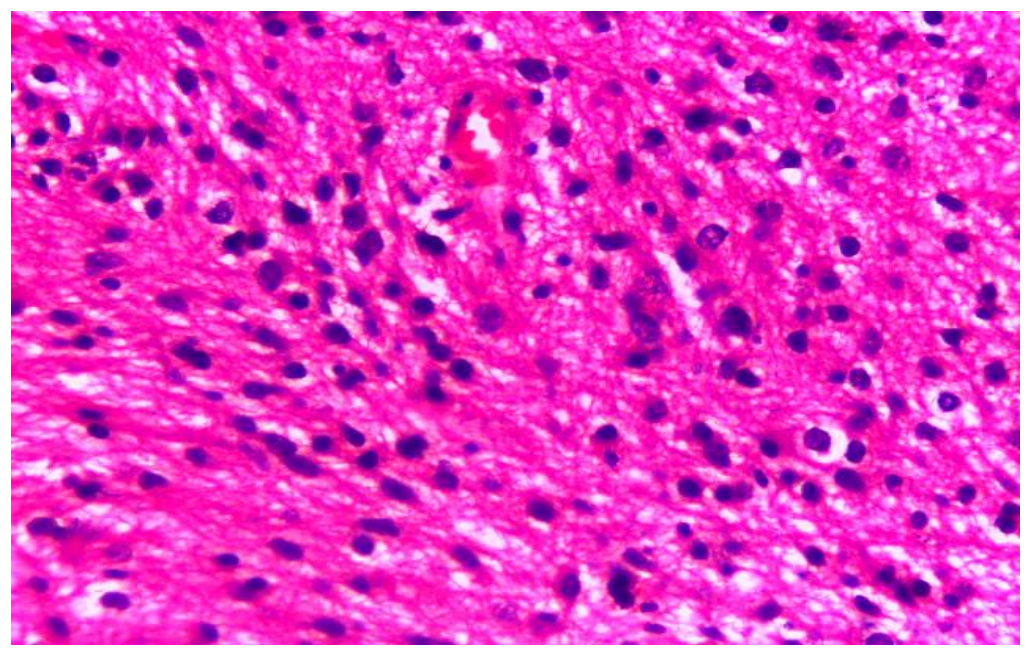

Figure (1) A case of anaplastic astrocytoma (grade III); High maginification showed increased cellularity, marked cellular pleomorphism and nuclear atypia (H\&Ex400).

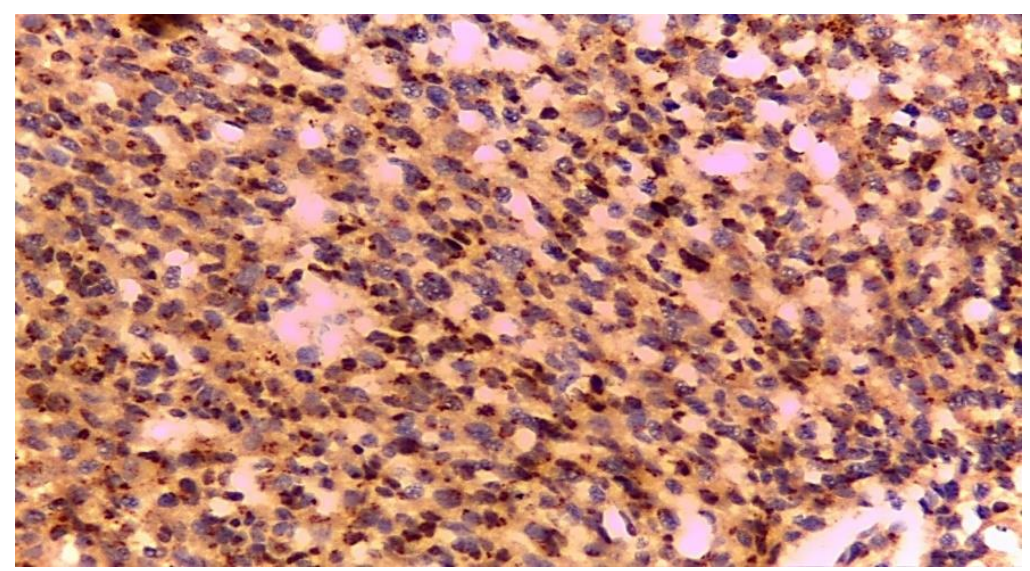

Figure (2): Anaplastic astrocytoma (grade III): The cells showed nuclear high Fli-1 score $=6$ (percentage score was 2) $\mathrm{x}$ (Intensity score was 3), (APCX400). 


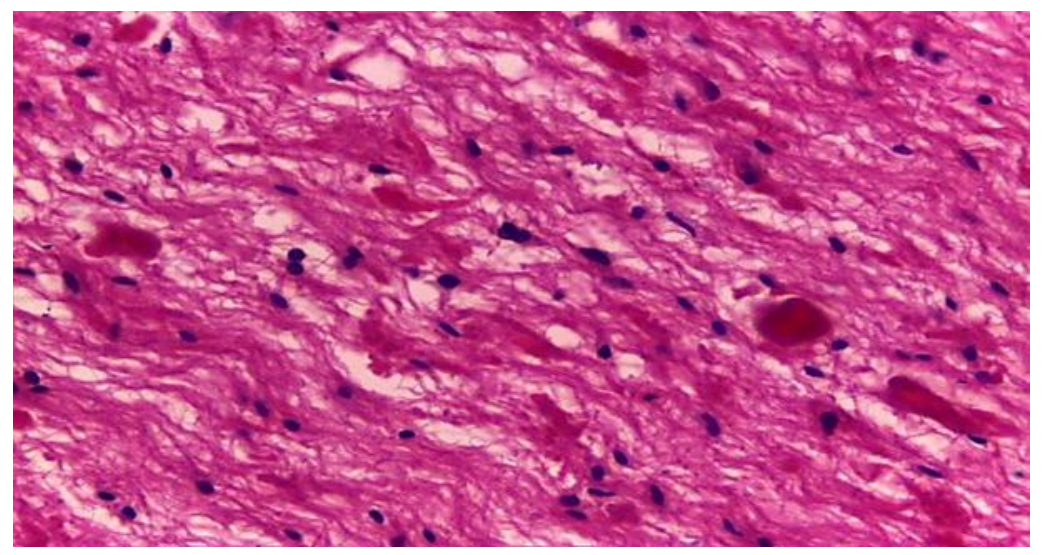

Figure (3) A case of pilocytic astrocytoma (grade I): High magnification showed numerous rosenthal fibers in a pilocytic astrocytoma. The rosenthal fibers appear as homogeneous, brightly eosinophilic, corkscrew-shaped structures $(\mathbf{H} \& \mathbf{E}$

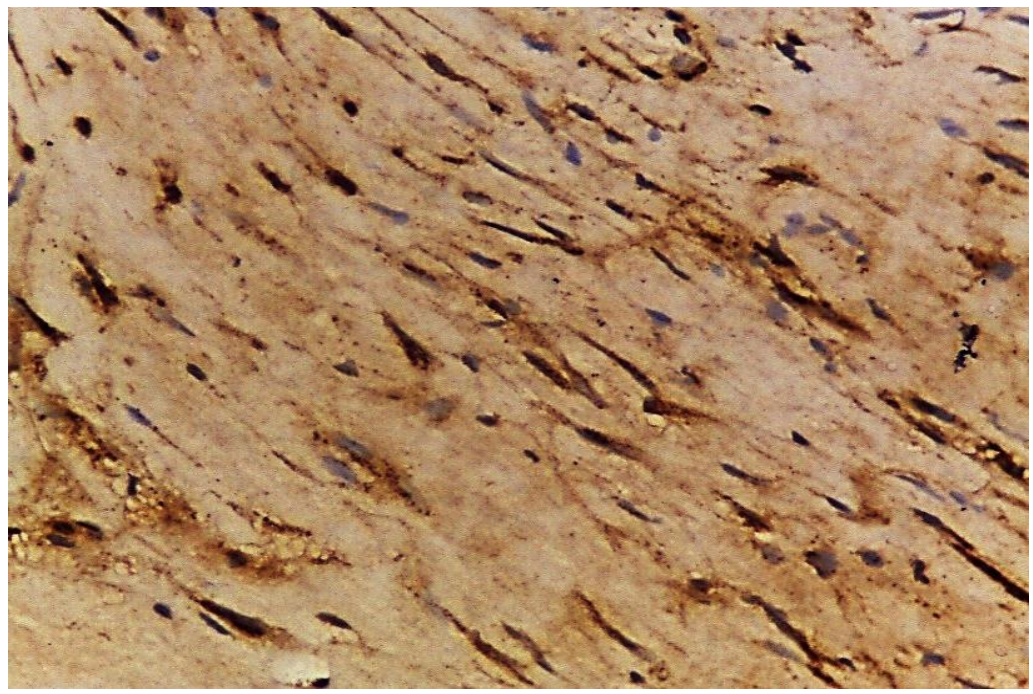

Figure (4): A case of pilocytic astrocytoma (grade I): The cells showed low nuclear Fli-1 score $=2$ (percentage score was 1 ) x (Intensity score was 2 ) (APCX400). 


\section{Discussion}

Overexpression of Fli-1 has a role in tumor prognosis in several malignancies and it may has a diagnostic and therapeutic role for astrocytoma. A positive statistical correlation between Fli-1 score and patient's survival was also detected in many other tumours as ovarian cancer (5), breast carcinoma (6), endometrial carcinoma (10), nasopharyngeal carcinoma (8) and nonsmall cell lung carcinoma (NSCLC) (11).

In this study, glioblastoma (grade IV) was the most common astrocytic tumour while grade I tumours (pilocytic astrocytoma and SEGA) were the least common astrocytic tumours. This was in line with a previous study (12) where it was found that glioblastoma (grade IV) was the most common astrocytic tumour and pilocytic astrocytoma (grade I) was the least common astrocytic tumour.

The current study showed $33.3 \%$ of cases were cerebral frontal, $44.4 \%$ of cases cerebral non frontal, $13.3 \%$ of cases in posterior fossa, $6.7 \%$ of cases in ventricles, and $2.3 \%$ of cases was in optic nerve. This study revealed that cerebral site (frontal and non-frontal) was the most common site for tumour. This was in agreement with studies carried before (13\& 12).

Regarding age distribution in our study, ages of astrocytoma cases ranged from 4 to 67 years old with age, mean \pm sd $(37$ years \pm 17.38). Maximum age group for astrocytoma cases (13.3\%) was in the seventh decade. The age groups were selected according to the Surveillance, Epidemiology, and End Results (SEER) program data base (1999-2010) $(14,16)$. This was close to the studies carried out before $(15 \& 13)$ where it was reported that the mean age of occurrence of astrocytoma was 37.77 years.

Regarding gender distribution in our study there was a male predominance. $60 \%$ of cases were males and $40 \%$ of cases were females with male to female ratio $1.5: 1$.This is close to the study performed previously (17 \& 13) which reported that the incidence of astrocytoma is higher in men than women with male to female ratio 1.4:1. They explained the male predominance that female hormones have preventive effects on tumorigenesis.

In this study $60 \%$ of cases showed high Fli -1 score and $40 \%$ of cases showed 
low Fli-1 score. . All grade III, IV cases had high Fli-1 score, while most of grade I, II cases had low Fli-1 score. The low grade cases that have high Fli-1 in this study showed recurrence.

In this study Roc curve analysis was done to measure sensitivity and specificity of Fli-1 in differentiation between low and high grade of astrocytoma using cutoff value >4. The ROC curve showed that the cutoff point $>2$ for Fli-1 score showed sensitivity of $95.65 \%$, specificity of $77.27 \%$ and area under curve (AUC) of $90.8 \%$ while the cutoff point $>4$ showed sensitivity of $81.5 \%$, specificity of $94.4 \%$ and AUC of $86.7 \%$ so the cutoff point $>2$ had the highest sensitivity and AUC.

In this study there was a statistical significant correlation between Fli-1 and age of studied cases ( $\mathrm{p}$ value <0.01). This in contrary to the study done on 2017 (6) where it was found that there was no statistically significant correlation between Fli-1 score and age of the studied cases (p value $<0.01)$. This could be explained that our study was including grade I astrocytic tumours not only grade II, III, IV as the study done (6) as grade I tumours mostly occur in young age.
In this study there was no statistically significant correlation between Fli-1 score and sex of the studied cases ( $p$ value $>0.05$ ). This was in agreement with the study (6) that found that there was no statistically significant correlation between Fli-1 score and sex of the studied cases with $\mathrm{p}$ value $>0.05$.

In our study there was statistically highly significant correlation found between score of Fli-1 and site of tumour in studied cases (p- value < 0.01). High Fli-1 score was detected significantly in tumours with cerebral site. This could be explained as high grade astrocytic tumours mostly occur in cerebral site.

In this study there was a statistically high significant direct correlation between Fli-1 score and grade of tumours in studied cases (p-value < 0.01). High grade cases had high Fli-1 score.

This was in line with many studies done by previously $(6,18, \& 19)$ that found statistically high significant direct correlation between Fli-1 score and Grade. They explained that Fli-1 is a nuclear transcription factor that involved in cellular proliferation and tumorigenesis as it 
activates VEGF leading to increased angiogenesis and malignant progression.

In this study there was a statistically significant direct correlation between Fli-1 score and size of tumor in studied patients with $\mathrm{p}$ value $<0.05$. High Fli-1 score was detected significantly in tumors with size $\geq$ $3 \mathrm{~cm}$.

This was in contrary to studies done (6), which found that there was no statistically significant correlation between Fli-1 score and size of tumour ( $\mathrm{p}$ value $<0.05)$.This could be explained as this study include grade I tumours (pilocytic and subependymal giant cell astrocytoma) which usually have small tumour size than other grades however studies (6) was only on grade II, III, IV.

In this study Kaplan Mayer analysis showed that there was statistically significant increase in the progression free and overall survival in cases with low Fli-1 score than those with high Fli-1 score with p-value $<0.01$ (Table 3,4)

This was in line with studies done previously $(10,8, \& 6)$ that found high level of Fli-1 associated with poor overall and progression free survival.
This was in line with other study (6), as Kaplan-Meier analysis and subsequent logrank analysis confirmed that high level of Fli-1 expression correlated significantly with poor overall survival $(P<0.01)$.

This was in agreement with a study carried before (18) that found that Fli-1 is highly expressed in glioblastoma (grade IV) with basal small levels in healthy tissues.

This study was close to other studies (20 \&21) that reported that Fli-1 is expressed have and directly implicated with astrocytoma cell proliferation, migration and invasion. It was also found that Fli-1 is overexpressed in glioblastoma cells promoting resistance to both radiation and drugs as temozolomide (19).

Fli -1 can act as either a transcriptional activator or a suppressor to regulate genes involved in cell proliferation, survival, or differentiation. Over expression of Fli-1 affects several cell signalling pathways, among which WNT, Pi3k-akt, and VEGF signalling pathways are significantly affected inducing genomic instability (22, 23).

Fli-1 negatively regulates the tumor suppressor p53 by directly binding to the promoter of MDM2 gene and stimulating of 
its transcription and up-regulating MDM2 destabilizing the anti-apoptotic protein p53. Fli-1 acts as oncogene by promoting proliferation and inhibiting p53 function. Downregulation of p53 accelerates tumor progression by inducing genomic instability. Fli-1 induces expression of anti-apoptotic genes B-cell lymphoma 2 (Bcl- 2) and Bcl-x $(24,25)$.

Tumor cells regulate angiogenesis through secretion of factors that stimulate neovascularization. Extracellular cells (ECs) express high levels of Fli-1. Fli-1 regulates angiogenesis by controlling expression of key angiogenic genes. Expression of Fli-1 in ECs activates endoglin during angiogenesis. Endoglin is upregulated in ECs to control cellular response to TGF- $\beta$. Fli-1 controls the recruitment and proliferation of smooth muscle cells and pericytes, stabilizing newly formed capillaries during angiogenesis (18).

\section{Conclusion}

Fli-1 had a correlation with grade of tumor and survival of cases in astrocytoma. It may have a diagnostic role in differentiation between low and high grade astrocytic tumors. It may have potential prognostic value in astrocytoma associated with aggressiveness of tumor and could be involved in progression of astrocytoma.

\section{References:}

1-Ostrom QT, Gittleman H , Jordan X, et al CBTRUS statistical report:primary brain and central nervous system tumors diagnosed in the United States in 2009-2013. Neuro- oncology. 2016; 18:v1-v75.

2-- Zalata KR, El-Tantawy DA, Abdel-Aziz A, et al Frequency of central nervous system tumors in delta region, Egypt. Indian J Pathol Microbiol. 2011. 54:299-306.

3-Yehia D, Fikry M, Gozlan S, et al MicroRNA Expression in Low- and High-Grade Gliomas in Pediatric Patients and Correlation with Matrix Metalloproteinase Expression. J Mol Genet Med. 2018 12: 370

4-Chourmouzi D, Papadopoulou E, Konstantinidis M, et al Manifestations of pilocytic astrocytoma: a pictorial review. Insights Imaging. 2014 Jun; 5(3):387-402.

5-Song W, Hu L, Li W, et al Oncogenic Fli-1 is a potential prognostic marker for the progression of epithelial ovarian cancer. BMC Cancer, 2014; 14:424

6-Tsai HP, Tsai TH, Hsieh YJ, et al. Overexpression of Fli-1 in astrocytoma is associated with poor prognosis. Oncotarget. 2017 Apr 25; 8(17):29174-29186.

7- Torlakovic EE, Slipicevic A, Florenes VA, et al. Fli-1 expression in malignant melanoma. Histol Histopathol. 2008; 23:1309-1314.

8- Liang X, Shi D, Yun J, et al. Friend leukemia virus integration 1 expression has prognostic significance in nasopharyngeal carcinoma. Transl Oncol. $2014 ; 7: 493-502$.

9- Scheiber MN, Watson PM, Rumboldt T, et al. FLI1 expression is correlated with breast cancer cellular growth, migration, and invasion and altered gene expression. Neoplasia. 2014; 16:801813

10-Song W, Zhang T, Li W, et al. Overexpression of Fli-1 is associated with adverse prognosis of Endometrial Cancer. Cancer Investig. 2015, 33, 469-475. 
11-Lin SF, Wu CC, Chai CY. Increased FLI-1 Expression is Associated With Poor Prognosis in Non-Small Cell Lung Cancers. Appl Immunohistochem Mol Morphol. 2016 Sep; 24(8):556-61.

12- Qaisrani, A., Shaheen, U., Nazir, S., et al. Morphological analysis of astrocytoma. Pakistan Journal of Physiology. 2020; 16(3), 22-24.

13- Lin, Z., Yang, R., Li, K.et al Establishment of age group classification for risk stratification in glioma patients. BMC Neurol. $2020 \mathrm{Aug}$ 20;20(1):310.

14- Alattar A A, Carroll, KT , Bryant A K., et al. Prognostic Importance of Age, Tumor Location, and Tumor Grade in Grade II Astrocytomas: An Integrated Analysis of the Cancer Genome Atlas and the Surveillance, Epidemiology, and End Results Database. World Neurosurg. 2019 Jan; 121:e411-e418.

15-Abu O, Rahman A, Khan R, et al. Magnetic Resonance Spectroscopy for Prediction of Grades of Diffusely Infiltrating Intracranial Astrocytomas. J Neurosci Rural Pract. 2020; 11(4):581-584.

16-Schupper AJ, Hirshman BR, Carroll KT, et al Effect of Gross Total Resection in World Health Organization Grade II Astrocytomas: SEER-Based Survival Analysis. World Neurosurg. 2017 Jul; 103:741-747.

17- Hirtz A, Rech F, Dubois-Pot-Schneider H, et al. Astrocytoma: A Hormone-Sensitive Tumor? International Journal of Molecular Sciences. 2020; 21(23):9114.
18-Miao B, Bauer AS, Hufnagel $K$, et al. The transcription factor FLI1 promotes cancer progression by affecting cell cycle regulation. Int J Cancer. 2020 Jul 1; 147(1):189-201

19- Rajesh Y, Biswas A, Banik P, et al. Transcriptional regulation of HSPB1 by Friend leukemia integration-1 factor modulates radiation and temozolomide resistance in glioblastoma. Oncotarget. 2020 Mar 31; 11(13):1097-1108.

20- Xu, Y., Gu, S., Bi, Y., et al. Transcription factor PU.1 is involved in the progression of glioma. Oncology Letters. 2018; 15, 3753-3759.

21-Babal YK, Kandemir B, Kurnaz IA. Gene Regulatory Network of ETS Domain Transcription Factors in Different Stages of Glioma. J Pers Med. 2021 Feb 17; 11(2):138.

22-Ma Y, Xu B, Yu J, et al Fli-1 Activation through Targeted Promoter Activity Regulation Using a Novel 3', 5'-diprenylated Chalcone Inhibits Growth and Metastasis of Prostate Cancer Cells. Int J Mol Sci. 2020 Mar 23; 21(6):2216.

23- Xie B, Hu F, Li M, et al FLI-1 mediates tumor suppressor function via Klotho signaling in regulating CRC. Cell Biol Int. 2020 Jul; 44(7):1514-1522.

24- Li Y, Luo H, Liu T, et al. The ets transcription factor Fli-1 in development, cancer and disease. Oncogene. 2015; 34(16):2022-2031.

25- Ota Fuchs (2017) "Importance and Presentation of Transcription Factor Fli-1 in Hematopoiesis and in Hematological and Other Malignancies" ARC Journal of Hematology. 2017; 2(2): 23-37

To cite this article: Hala A. Agina, Ahlam A. Abdelmaksoud, Eman S. Omar, Marwa S. Abdallah, Shimaa G. Ahmed. The Significance of Friend Leukemia Virus Integration 1Transcription Factor (Fli-1) in Astrocytoma. BMFJ 2022; 39 (academic issue): $153-167$. 